\title{
Decentralized Control of a Nine-phase Permanent Magnet Generator for Offshore Wind Turbines
}

\author{
Eduardo Prieto-Araujo, Student Member, IEEE, Adrià Junyent-Ferré, Member, IEEE, David Lavèrnia-Ferrer \\ and Oriol Gomis-Bellmunt, Senior Member, IEEE
}

\begin{abstract}
This article presents a decentralized current control approach for a nine-phase wind turbine generator. This type of generator has three different three-phase stators sharing the same machine yoke, connected to the grid by means of three different Voltage Source (VS) back-to-back power converters. Due to the machine configuration, magnetic couplings are present between the three stators, complicating the design and implementation of the machine current controllers. Rather than a centralized control approach, this paper proposes a methodology to design a decentralized machine control to regulate the active and reactive power flowing through each stator independently. A complete dynamic analysis is performed in order to design the controller to reduce the coupling effects within the machine while ensuring a proper dynamic performance. The control strategy is validated through simulation and experimental results.
\end{abstract}

Index Terms

Permanent magnet machines, multiphase variable-speed drives, decentralized control, wind energy.

\section{INTRODUCTION}

$\mathbf{O}$ FFSHORE wind power is one of the most promising renewable energies [1]. In the North Sea, a number of wind power plants will be commissioned in the next years. Offshore wind has advantages compared to onshore wind, such as abundant wind resources [2], no size limitation of the turbines and a reduced visual impact on population. On the other hand, offshore installations have some financial and technical challenges [3] such as long distance transmission systems required for the energy grid integration, the complex and expensive installation, operation and maintenance tasks, besides the limitations in footprint and weight of the offshore structures. Therefore, offshore turbines must be designed to be highly efficient, light and reliable [4], [5], with reduced maintenance requirements, in order to increase the cost-effectiveness of the system. One of the most interesting topologies proposed for offshore wind is the direct driven multi-pole Permanent Magnet Synchronous Machine (PMSM) with Full Power Converter (FPC) [5], a turbine concept which does not include the gearbox. Based on this idea, different advanced generator concepts as the direct driven multi-pole triple three-phase stator PMSM have been proposed for wind energy [6], [7], [8].

The aforementioned topology expands the operational possibilities of the conventional three-phase machines. Multiphase machines have interesting advantages [9] such as the reduction of the grid converter power electronics ratings and improved fault tolerance capabilities due to the redundant structure. Specifically, this machine configuration allows operating each threephase system independently from the other two, considering that each stator is connected to the grid by means of a FPC. The active and reactive current flowing through each stator can be regulated to be operated at different power levels, even enabling to operate with two or one stators [7]. However, the design of the current controller required to operate a nine-phase machine is not straightforward due to the machine internal magnetic couplings between stator phases.

Different approaches to control the multi-star machine currents have been proposed in the literature [9]. The classical vector control can be extended for multiphase machines [9] considering that each FPC converter is able to regulate its corresponding three-phase stator current, even including a centralized controller able to decouple the effects between stators [10], [11], [12]. In order to apply this decoupling, fast communications between converter controllers or a centralized control is required. However, in this work the nine-phase machine control is designed to be performed by local stator controllers [13], without communications between them, posing the challenge of handling the stator coupling interaction.

The proposed strategy can be interesting for applications that do not include communications among the different converters connected to each PMSM stator. If fast communications between converters or a centralized controller are available, control approaches able to decouple the interaction between stators may be implemented [10], [11], [12], due to their notable performance. However, if these communications fail or the bandwidth is not high enough to compensate the stator couplings, the system could be still controlled with the proposed decentralized strategy, ensuring a proper behavior.

For wind power multi-star generators, the decentralized control methodology can be interesting as the machine can still be operated, even in case of a communication failure between the stator controllers. This problem becomes specially important for offshore wind power plants where maintenance tasks are more complicated compared to onshore sites, due to limited accessibility [4], and failures could last for a larger period of time. Thus, this control strategy could avoid the disconnection of an offshore machine that otherwise could remain non-operative for a long period of time, with the corresponding negative economic impact. 
To the best of the authors knowledge, there is no methodology for designing the decentralized vector control of a triple three-phase PMSM, without communications among the different converter local current controllers, that ensures a proper machine performance, limiting the interaction between the different stator currents. This work describes the procedure to design a decentralized current control for a direct driven triple three-phase PMSM. The active and reactive power flowing through each of the machine stators are regulated through six different controllers, implementing two of them at each of the three-phase converters. The main issue during the design of the controllers is the existing coupling between the machine phases. A detailed machine model is obtained assuming non-zero mutual coupling between the three stators in order to analyze the existing interactions. Once the model is derived, the machine current regulators are designed based on the machine open and closed loop frequency response of the multi-variable system. The control objectives defined are the current set-points tracking with a proper dynamic performance and a reduced interaction between stators during transients. The proposed decentralized controller is validated through dynamic simulations and implemented in a real test rig including a scaled $30 \mathrm{~kW}$ nine-phase PMSM.

The paper is organized as follows. The equations of a generic triple three-phase machine model are obtained in Section II. These equations are used in Section III to define the methodology to design the decentralized current controllers. In Section $\mathrm{IV}$, this methodology is applied to design the control of a triple three-phase wind turbine generator and in Section V simulation results of the proposed controller are shown. Finally, in Section VI experimental results of the operation of a scaled nine-phase wind turbine generator based on the proposed decentralized control are presented.

\section{MACHINE MODELING}

This section details the nine-phase PMSM model derivation in the reference frame fixed in the rotor. Multi-phase machine modeling in the conventional $q d$ reference frame has been previously addressed in [14] for an induction machine with multiple winding sets. Among the different multi-phase machines topologies, the dual-stator induction machine has been widely analyzed in the literature [9], [10], [15], [16]. Regarding dual-stator PMSMs, different modeling approaches can be found in [17], [18], even for wind generation systems in [19]. Also, specific modeling techniques for nine-phase PMSMs have been proposed [6], [7]. For the present study, the following assumptions are made to obtain the model:

- Nine-phase, triple three-phase stator configuration.

- Generic winding configuration.

- Round rotor machine. The inductances are considered to be independent from the rotor position [14].

- Saturation effects are not considered.

Next, the voltage equations of the nine-phase machine are presented. The electrical phase layout of the machine stators (s1, s2, s3) is shown in Fig. 1.

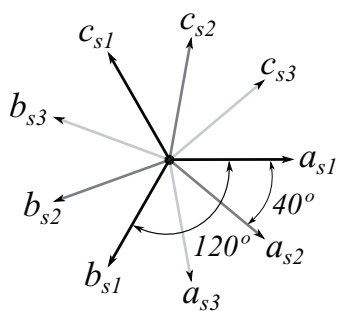

Fig. 1. Machine electrical phase distribution

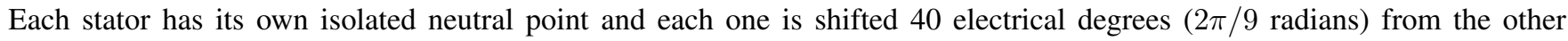
two. Phases $a_{s 1}, b_{s 1}, c_{s 1}, a_{s 2}, b_{s 2}, c_{s 2}$ and $a_{s 3}, b_{s 3}, c_{s 3}$ form stators 1,2 and 3 respectively. The machine voltage equations can be defined as [14]:

$$
v_{s}^{\overline{a b c}}=\mathbf{R}_{\mathbf{s}} i_{s}^{\overline{a b c}}+\frac{d}{d t} \lambda_{s}^{\overline{a b c}}
$$

where $v_{s}^{\overline{a b c}}$ is the vector of voltages applied to the machine by the converters, $i_{s}^{\overline{a b c}}$ is the vector of currents flowing through the machine, $\lambda_{s}^{\overline{a b c}}$ is the flux linkage vector of the stator windings and $\mathbf{R}_{\mathbf{s}}$ is the diagonal matrix that represents the resistance $R$ of the machine windings. The dimension of these vectors is $9 \times 1$ and internally, the variables are sorted as:

$$
x=\left(x_{s 1}^{a}, x_{s 2}^{a}, x_{s 3}^{a}, x_{s 1}^{b}, x_{s 2}^{b}, x_{s 3}^{b}, x_{s 1}^{c}, x_{s 2}^{c}, x_{s 3}^{c}\right)^{T}
$$

where $x$ is either a current, voltage or flux magnitude, $a, b$ and $c$ are the phases and $s_{i}$ the corresponding stator. The flux linkage term $\lambda_{s}^{a b c}$ can be expanded as:

$$
\lambda_{s}^{\overline{a b c}}=\mathbf{L}_{\mathbf{s}} i_{s}^{\overline{a b c}}+\lambda_{m}\left(\begin{array}{c}
\sin (\theta) \\
\sin (\theta-\varphi) \\
\vdots \\
\sin (\theta-8 \varphi)
\end{array}\right)
$$


where $\mathbf{L}_{\mathbf{s}}$ is the inductance matrix of the machine, $\lambda_{m}$ is the flux linkage of the magnets, $\varphi$ is the electrical angle between two phases (in this case 40 degrees) and $\theta$ is the electrical angle of the machine. It is assumed that the flux created by the magnets is sinusoidally distributed along the air-gap. Specifically, the inductance matrix $\mathbf{L}_{\mathbf{s}}$ of a nine-phase machine, considering a generic winding distribution, can be expressed as [6], [7]:

$$
\mathbf{L}_{\mathbf{s}}=\left(\begin{array}{ccccccccc}
L & M_{1} & M_{2} & M_{3} & M_{4} & M_{4} & M_{3} & M_{2} & M_{1} \\
M_{1} & L & M_{1} & M_{2} & M_{3} & M_{4} & M_{4} & M_{3} & M_{2} \\
M_{2} & M_{1} & L & M_{1} & M_{2} & M_{3} & M_{4} & M_{4} & M_{3} \\
M_{3} & M_{2} & M_{1} & L & M_{1} & M_{2} & M_{3} & M_{4} & M_{4} \\
M_{4} & M_{3} & M_{2} & M_{1} & L & M_{1} & M_{2} & M_{3} & M_{4} \\
M_{4} & M_{4} & M_{3} & M_{2} & M_{1} & L & M_{1} & M_{2} & M_{3} \\
M_{3} & M_{4} & M_{4} & M_{3} & M_{2} & M_{1} & L & M_{1} & M_{2} \\
M_{2} & M_{3} & M_{4} & M_{4} & M_{3} & M_{2} & M_{1} & L & M_{1} \\
M_{1} & M_{2} & M_{3} & M_{4} & M_{4} & M_{3} & M_{2} & M_{1} & L
\end{array}\right)
$$

The inductance terms of the matrix can be analytically calculated [18], [20], [21] for a specific winding. Alternatively, if detailed information about the geometry is available, Finite Element Models (FEM) can be used to calculate the numerical value of the inductances. The correlation of the machine inductance values is depicted in Fig. 2.

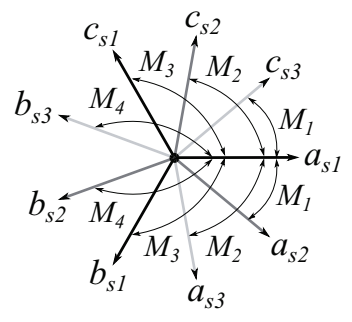

Fig. 2. Inductance values phase relations

Finally, to obtain the complete voltage equations of the machine, the flux term (3) is substituted into (1):

$$
v_{s}^{\overline{a b c}}=\mathbf{R}_{\mathbf{s}} i_{s}^{\overline{a b c}}+\mathbf{L}_{\mathbf{s}} \frac{d}{d t} i_{s}^{\overline{a b c}}+\lambda_{m} \omega\left(\begin{array}{c}
\cos (\theta) \\
\cos (\theta-\varphi) \\
\vdots \\
\cos (\theta-8 \varphi)
\end{array}\right)
$$

where $\omega$ is the electrical rotational speed of the machine.

\section{DECENTRALIZED CONTROL OF THE MACHINE}

In this section, the decentralized current controller is designed analyzing the obtained machine voltage differential equations. The main control objectives are:

- Stator independent active and reactive power regulation.

- Ensure a time response performance for an active or reactive current reference change.

- Minimize the interactions between stators.

\section{A. Machine equations in the rotating reference frame}

In order to simplify the control problem, the well-known Park variable transformation [22] is applied to each of machine stator equations, referenced to the electrical rotation angle $\theta$ :

$$
\mathbf{T}(\theta)=\frac{2}{3}\left(\begin{array}{ccc}
\cos (\theta) & \cos \left(\theta-\frac{2 \pi}{3}\right) & \cos \left(\theta+\frac{2 \pi}{3}\right) \\
\sin (\theta) & \sin \left(\theta-\frac{2 \pi}{3}\right) & \sin \left(\theta+\frac{2 \pi}{3}\right) \\
\frac{1}{2} & \frac{1}{2} & \frac{1}{2}
\end{array}\right)
$$

This transformation is extended for three stators, considering the electrical angle displacement between them [11], [23]:

$$
\mathbf{T}^{\overline{q d}}(\theta)=\frac{2}{3}\left(\begin{array}{ccc}
\mathbf{T}(\theta) & \mathbf{0}_{\mathbf{3 \times 3} 3} & \mathbf{0}_{\mathbf{3 \times 3}} \\
\mathbf{0}_{\mathbf{3 \times 3}} & \mathbf{T}(\theta-\varphi) & \mathbf{0}_{\mathbf{3 \times 3}} \\
\mathbf{0}_{\mathbf{3 \times 3}} & \mathbf{0}_{\mathbf{3 \times 3}} & \mathbf{T}(\theta-2 \varphi)
\end{array}\right)
$$

being each component of (7) a $3 \times 3$ matrix dimension. Note that this transformation is applied for vectors sorted as:

$$
x=\left(x_{s 1}^{a}, x_{s 1}^{b}, x_{s 1}^{c}, x_{s 2}^{a}, x_{s 2}^{b}, x_{s 2}^{c}, x_{s 3}^{a}, x_{s 3}^{b}, x_{s 3}^{c}\right)^{T}
$$


Then, in order to properly apply the transformation to the variables sorted as shown in (2), the elements of (7) should be rearranged. Reordering and applying (7) to the machine equations (5), the following system is obtained:

$$
v_{s}^{\overline{q d}}=\left(\mathbf{R}_{\mathbf{s}}+\mathbf{A}_{\mathbf{1}}\right) i_{s}^{\overline{q d}}+\mathbf{A}_{\mathbf{2}} \frac{d}{d t} i_{s}^{\overline{q d}}+v_{m}^{\overline{q d}}
$$

where $v_{s}^{\overline{q d}}, v_{m}^{\overline{q d}}$ and $i_{s}^{\overline{q d}}$ are the converter voltages applied to the machine, the machine voltages due to the magnet flux linkages and the current flowing through the machine, respectively, in the new reference frame. The generic variable arrangement is:

$$
x_{s}^{\overline{q d}}=\left(x_{s 1}^{q}, x_{s 2}^{q}, x_{s 3}^{q}, x_{s 1}^{d}, x_{s 2}^{d}, x_{s 3}^{d}\right)^{T}
$$

Note that the number of equations has been reduced from nine to six because the machine windings have an isolated neutral point for each stator; thus, zero sequence currents flowing through the machine are null. Specifically, matrices $\mathbf{A}_{\mathbf{1}}$ and $\mathbf{A}_{\mathbf{2}}$ in (9) show the relations between the variables of the machine in the new reference frame:

$$
\begin{aligned}
\mathbf{A}_{\mathbf{1}}=\omega & \left(\begin{array}{cccccc}
0 & -C & C & A & B & B \\
C & 0 & -C & B & A & B \\
-C & C & 0 & B & B & A \\
-A & -B & -B & 0 & -C & C \\
-B & -A & -B & C & 0 & -C \\
-B & -B & -A & -C & C & 0
\end{array}\right) \\
\mathbf{A}_{2} & =\left(\begin{array}{cccccc}
A & B & B & 0 & C & -C \\
B & A & B & -C & 0 & C \\
B & B & A & C & -C & 0 \\
0 & -C & C & A & B & B \\
C & 0 & -C & B & A & B \\
-C & C & 0 & B & B & A
\end{array}\right) \\
B & =-\cos \frac{\pi}{9} M_{4}-\cos \frac{5 \pi}{9} M_{2}+\cos \frac{2 \pi}{9} M_{1} \\
C & =-\cos \frac{7 \pi}{18} M_{4}+\cos \frac{\pi}{18} M_{2}-\sin \frac{2 \pi}{9} M_{1}
\end{aligned}
$$

Finally, the machine voltages vector, due to the magnet flux linkages, can be expanded as:

$$
v_{m}^{\overline{q d}}=\lambda_{m} \omega\left(\begin{array}{llllll}
1 & 1 & 1 & 0 & 0 & 0
\end{array}\right)^{T}
$$

Besides, the torque equation of the machine can also be obtained in the new reference frame:

$$
T_{m}=\frac{3}{2} \lambda_{m} P\left(i_{s 1}^{q}+i_{s 2}^{q}+i_{s 3}^{q}\right)
$$

where $P$ is the number of machine pole pairs. Hence, (13) shows that the machine torque only depends on the current $q$ variables, considered from now on as the active current components. On the other hand, the current $d$ variables are considered reactive components, as they do not contribute to the machine torque. There is no reluctance torque component due to the round rotor machine configuration.

\section{B. Decentralized vector control}

The decentralized control design is based on the classical vector control. It is designed considering that no communications are available between stators, thus three different vector controllers are designed, one for each stator. To do so, the equations for one stator are extracted from the total machine model (9), considering only the variables of the same stator, painted in gray in (11):

$$
\begin{aligned}
\left(\begin{array}{c}
v_{s i}^{q} \\
v_{s i}^{d}
\end{array}\right)=\left(\begin{array}{cc}
R & \omega\left(L-M_{3}\right) \\
-\omega\left(L-M_{3}\right) & R
\end{array}\right)\left(\begin{array}{c}
i_{s i}^{q} \\
i_{s i}^{d}
\end{array}\right)+ & \left(\begin{array}{cc}
L-M_{3} & 0 \\
0 & L-M_{3}
\end{array}\right) \frac{d}{d t}\left(\begin{array}{c}
i_{s i}^{q} \\
i_{s i}^{d}
\end{array}\right)+\lambda_{m} \omega\left(\begin{array}{c}
1 \\
0
\end{array}\right)+\left(\begin{array}{c}
d_{q} \\
d_{d}
\end{array}\right)
\end{aligned}
$$

where $d_{q}$ and $d_{d}$ gather the interaction elements between stators. The controller approach considers $d_{q}$ and $d_{d}$ to be exogenous signals or disturbances for the controller and they are not considered within this design stage. Then, the corresponding equations of one of the machine stators (14) are equivalent to a conventional three-phase machine. Thus, the classical design vector control 
can be employed [24], including the decoupling loop between the $q$ and $d$ variables. Hence, the first design step consists of applying the following variable change to the equations (14):

$$
\left(\begin{array}{c}
v_{s i}^{q} \\
v_{s i}^{d}
\end{array}\right)=\left(\begin{array}{c}
\hat{v}_{s i}^{q}+\omega\left(L-M_{3}\right) i_{s i}^{d}+\lambda_{m} \omega \\
\hat{v}_{s i}^{d}-\omega\left(L-M_{3}\right) i_{s i}^{q}
\end{array}\right)
$$

Substituting (15) in (14), decouples the machine $q$ and $d$ variables:

$$
\left(\begin{array}{l}
\hat{v}_{s i}^{q} \\
\hat{v}_{s i}^{d}
\end{array}\right)=\left(\begin{array}{cc}
R & 0 \\
0 & R
\end{array}\right)\left(\begin{array}{c}
i_{s i}^{q} \\
i_{s i}^{d}
\end{array}\right)+
$$

$$
\left(\begin{array}{cc}
L-M_{3} & 0 \\
0 & L-M_{3}
\end{array}\right) \frac{d}{d t}\left(\begin{array}{c}
i_{s i}^{q} \\
i_{s i}^{d}
\end{array}\right)
$$

Once $q$ and $d$ variables are decoupled, two Single Input Single Output (SISO) transfer functions in the Laplace domain can be found:

$$
\frac{I_{s i}^{q}(s)}{\hat{V}_{s i}^{q}(s)}=\frac{1}{\left(L-M_{3}\right) s+R} ; \quad \frac{I_{s i}^{d}(s)}{\hat{V}_{s i}^{d}(s)}=\frac{1}{\left(L-M_{3}\right) s+R}
$$

where $I_{s i}^{q}, I_{s i}^{d}, \hat{V}_{s i}^{q}$ and $\hat{V}_{s i}^{d}$ are the Laplace variables for the $q$ and $d$ voltages and currents of the stator $s_{i}$. Based on the plants obtained, two different controllers are designed, one for each corresponding current, based on the Internal Model Control (IMC) design methodology [24], [25]. This technique allows obtaining a controller that cancels the plant internal dynamics, while imposing a desired one. Applying this technique to the two system transfer functions (17), aiming to obtain a first order time response for the closed loop system, the controller to be implemented results in a conventional PI:

$$
K_{c}=\frac{K_{p} s+K_{i}}{s}
$$

with the following parameters:

$$
K_{p}=\frac{L-M_{3}}{\tau} ; \quad K_{i}=\frac{R}{\tau}
$$

where $\tau$ is the inverse of the first order time constant of the closed loop system. In order to control the complete machine, two different PI controllers per stator are required with their corresponding decoupling loops between $q$ and $d$ variables (15), as it is shown in Fig. 3. To complete the control design, the closed loop response time constant $\tau$ should be selected.

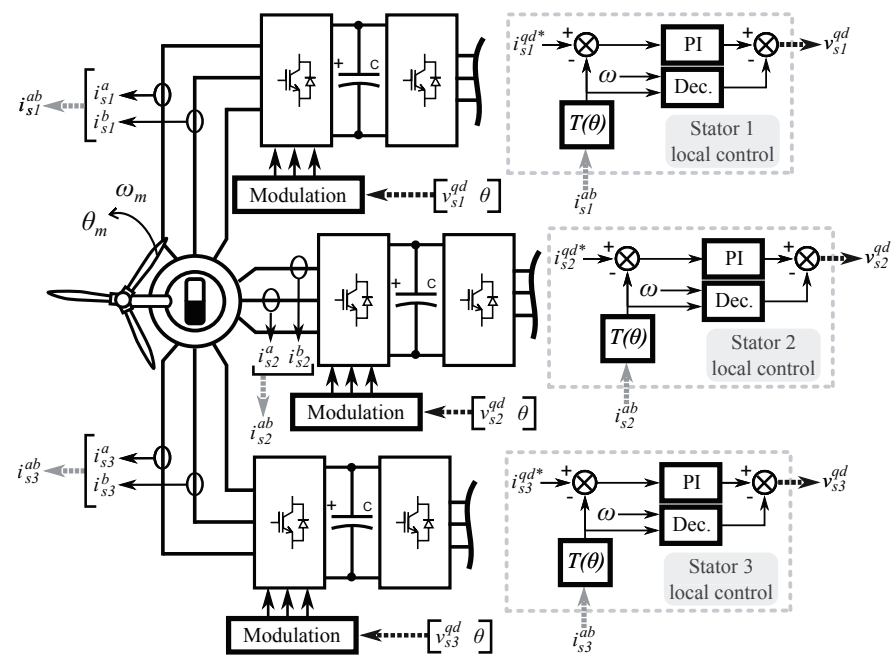

Fig. 3. Wind turbine nine-phase generator control

\section{Dynamic analysis and interaction between stators}

The controllers bandwidth is strictly related with the effect that a current change in one of the stators has over the currents in other stators. Then, a multi-variable frequency analysis is performed to the machine control system (Fig. 4), to properly select the controller closed loop time response to achieve a good control performance, while maintaining the effects between stators under certain boundaries. The control design steps are detailed next: 


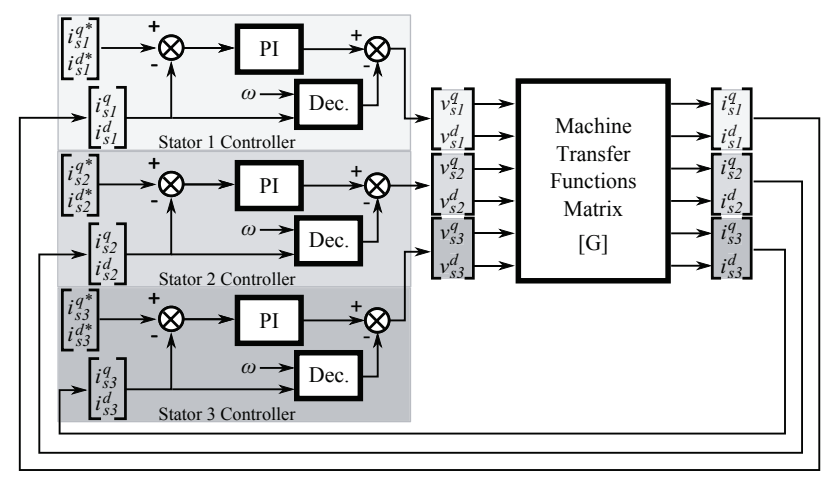

Fig. 4. Closed loop control of the multi-variable control system. Inputs: Current set-points in $q d$ reference, Outputs: machine currents in $q d$ reference

1) Open loop analysis: First, the open loop transfer function matrices of the machine is obtained (Matrix G in Fig. 4), in order to analyze the dynamics of the machine before including any controller. This transfer function matrix can be calculated as:

$$
\mathbf{G}=\mathbf{C}_{\mathbf{s}}\left(s \mathbf{I}_{\mathbf{6 x 6}}-\mathbf{A}_{\mathbf{s}}\right)^{-1} \mathbf{B}_{\mathbf{s}}
$$

where $\mathbf{I}_{6 \times 6}$ is a $6 \times 6$ identity matrix and $\mathbf{A}_{\mathbf{s}}, \mathbf{B}_{\mathbf{s}}$ and $\mathbf{C}_{\mathbf{s}}$ are the state-space matrices of the machine model, that can be obtained by isolating the derivatives of the current from the machine equations (9). Note that, open loop equations (9)-(11) show that the machine model is dependent on the rotational speed. Therefore, the open loop frequency analysis of the machine must be performed for different rotational speeds. Once the frequency response is obtained, large frequency gains that could cause interaction between stators, should be identified. The open loop transfer function matrix $\mathbf{G}$ has six voltage inputs and six current outputs (Fig. 4), hence thirty-six different transfer functions. Due to the symmetry of the machine model, only one voltage input (considering all current outputs) should be analyzed, because the frequency response is analogous for all the other inputs.

2) Closed loop analysis: Once the open loop analysis is completed, the frequency response of the closed loop multi-variable system is carried out, based on the closed loop transfer function matrix $\mathbf{T}$. This matrix can be obtained as:

$$
\mathbf{T}=\mathbf{G K}\left(\mathbf{I}_{\mathbf{6 x 6}}+\mathbf{G K}\right)^{-1}
$$

where $\mathbf{K}$ is a diagonal matrix with the designed PI controllers (18) in each component. The frequency response of the $\mathbf{T}$ matrix shows the dynamics between the current set-points $i_{s i}^{q *}$ and $i_{s i}^{d *}$ and the actual machine currents $i_{s i}^{q}$ and $i_{s i}^{d}$ in closed loop. Through the frequency analysis of the $\mathbf{T}$ matrix, the dynamics of the decentralized controller can be evaluated in closed loop, considering the following objectives:

- The current set-points $i_{s i}^{q *}$ and $i_{s i}^{d *}$ should be tracked without steady state errors, ensuring an approximate first order system response.

- Limit the error of the local current controllers, caused by current changes in the other stators. The closed loop matrix $\mathbf{T}$ relates the set-points $i_{s i}^{q d *}$ with the machine actual currents $i_{s i}^{q d}$. Then, setting a maximum allowed gain on the frequency representation of $\mathbf{T}$, the dynamic interaction between the different closed loop controllers is limited. In other words, the gain limitation can be understood as the maximum current deviation allowed in one stator current controller caused by a reference change $\Delta i_{s i}^{q d *}$ introduced in the current controller of another stator.

It is also interesting to plot the matrix $\mathbf{T}$ frequency response graph for different rotational speeds, due to the effect that the speed has on the machine behavior. Note also that, as in the open loop study, the frequency analysis of the $\mathbf{T}$ matrix is performed for a single set-point input (considering all the current outputs), due to the model symmetry.

\section{Grid side converter control}

In order to perform the machine control, the machine side converter require a controlled DC bus voltage to be able apply voltages to the corresponding stator. The mentioned DC voltage regulation is carried out, by each of the GSCs, as it is shown in Fig. 5. The output of the DC regulator corresponds to the input for the active current set-point of a classical vector control [24] carried out in the synchronous reference frame, defined by the grid angle obtained through a Phase Locked Loop (PLL) system [26]. Note that the control of the different GSCs do not interfere in the decentralized control approach, as long as it is operated properly. 


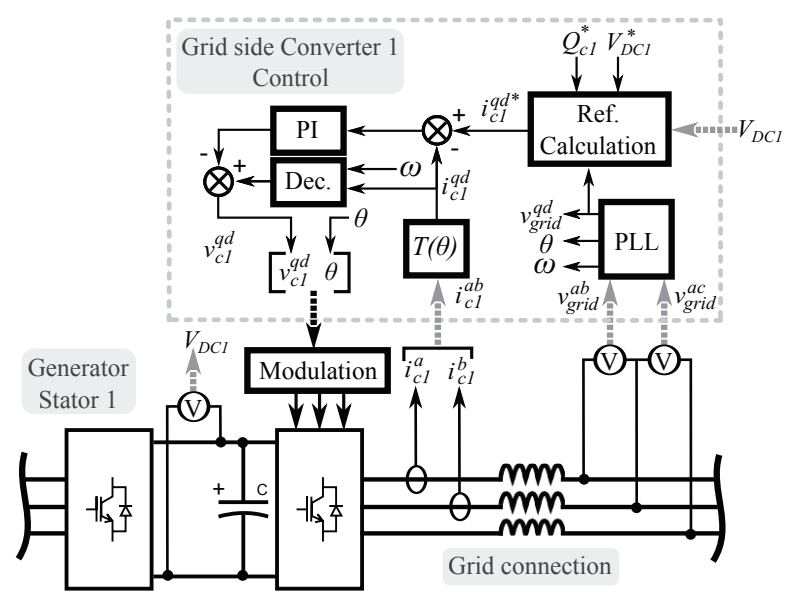

Fig. 5. Wind turbine grid side converters control

TABLE I

NINE PHASE GENERATOR PARAMETERS

\begin{tabular}{c|c|c}
\hline Parameter & Value & Units \\
\hline Inductance $L$ & 1.73 & $\mathrm{mH}$ \\
Inductance $M_{1}$ & -0.121 & $\mathrm{mH}$ \\
Inductance $M_{2}$ & -0.036 & $\mathrm{mH}$ \\
Inductance $M_{3}$ & -0.052 & $\mathrm{mH}$ \\
Inductance $M_{4}$ & -0.346 & $\mathrm{mH}$ \\
Phase resistance $R$ & 10 & $\mathrm{~m} \Omega$ \\
\hline Rated Phase voltage $V_{a n}$ & 550 & $\mathrm{~V}$ \\
Pole pairs $P$ & 60 & - \\
Rated mechanical speed $\omega_{n}$ & 11 & $\mathrm{~min}^{-1}$ \\
\hline
\end{tabular}

\section{CASE STUDY}

In this section, the methodology to design the decentralized control of the machine is applied to a specific triple three-phase wind generator. The characteristics are summarized in Table I.

First, substituting values from Table I into the machine model equations the open loop $\mathbf{G}$ transfer function matrix is obtained. The input selected for plotting the Bode diagram is the stator $1 q$ axis voltage $v_{s 1}^{q}$ and as outputs all the machine currents $i_{s i}^{q}$ and $i_{s i}^{d}$, obtaining six different curves. This diagram is obtained for four machine speeds: rated speed $\omega_{n}, 2 / 3$ and $1 / 3$ of the rated speed and zero speed (Fig. 6).
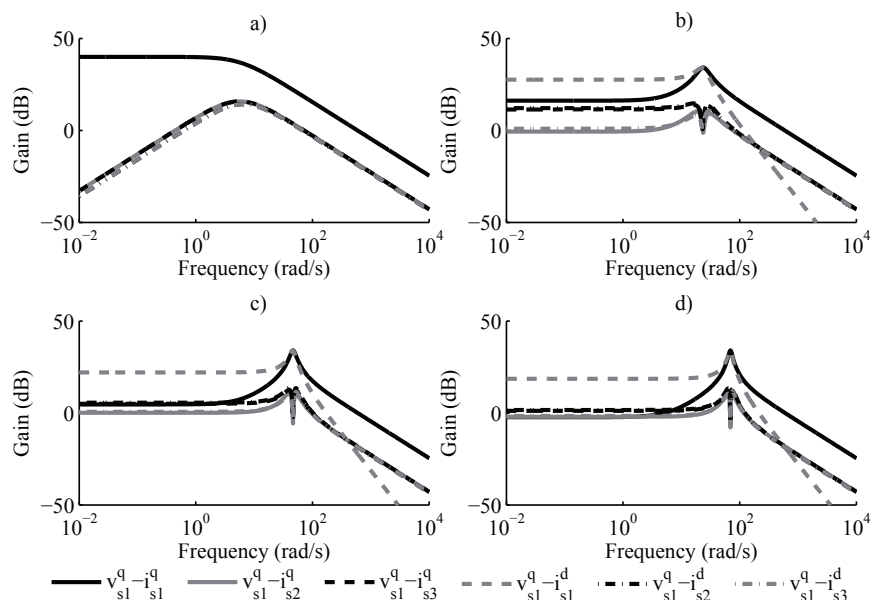

Fig. 6. Open loop transfer function matrix Bode diagram representation at different rotational speeds. Input: stator $1 q$ axis voltage, Outputs: machine currents in $q d$ reference

The open loop Bode diagram shows that the influence that the input voltage $v_{s 1}^{q}$ has over the current $i_{s 1}^{d}$ is strong at all rotational speeds, due to the existing coupling between $q$ and $d$ variables (14), except at very low speeds. This fact reinforces the requirement of the decoupling loop within each of the three-phase vector controllers. Moreover, cases b, $c$ and $d$ show large gains close to the rotational speed at which the Bode diagram is calculated. 
Following the discussion of the open loop system, the closed loop transfer function matrix $\mathbf{T}$ is computed based on the decentralized control approach employing six different PI controllers (18)-(19), leaving the closed loop time constant undefined. In order to properly select this constant, the frequency dynamic analysis of the closed loop transfer function $\mathbf{T}$ is performed. The Bode diagram representation, considering as input the stator $1 q$ axis current set-point $i_{s 1}^{q *}$ and as outputs all the machine currents $i_{s i}^{d}$ and $i_{s i}^{d}$, is plotted for different closed loop time responses of the controller: 100, 50, 20 and 10 ms. This procedure is repeated for different machine rotational speed values as it is shown in Figs. 7, 8, 9 and 10. In order to limit the coupling effect between stators, it is considered that a set-point $i_{s 1}^{q *}$ amplitude change $\Delta i_{s 1}^{q *}$ should deviate the current from the other stators $\left(i_{s i}^{q}\right.$ and $i_{s i}^{d}$, for $\left.\mathrm{i}=2,3\right)$, less than a $10 \%$ of the amplitude $\Delta i_{s 1}^{q *}$. Then, the corresponding gain limitation is:

$$
G_{l i m}=20 \log _{10}(0.1)=-20 \mathrm{~dB}
$$
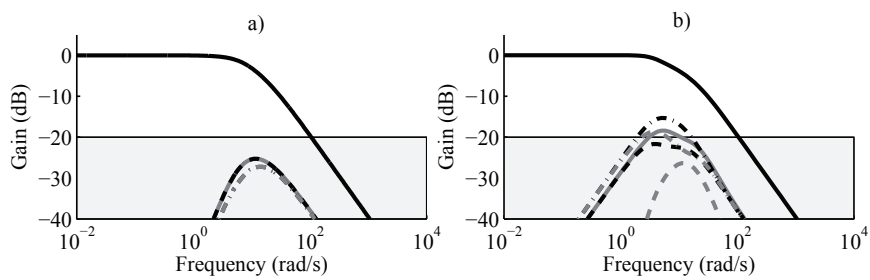

c)
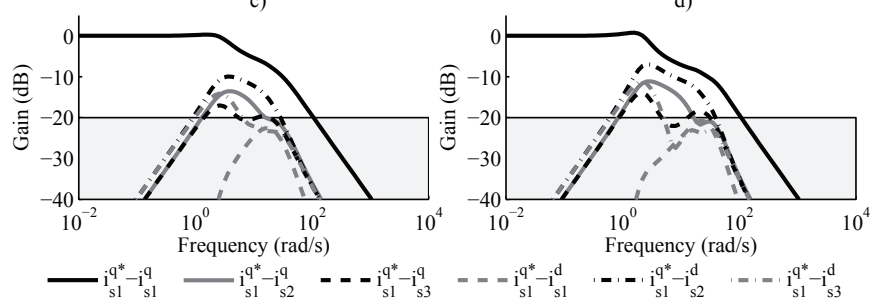

Fig. 7. Closed loop transfer function matrix Bode diagram representation at different rotational speeds. Input: stator $1 q$ axis current set-point. Outputs: machine currents in $q d$ reference. Closed loop time response design: $100 \mathrm{~ms}$
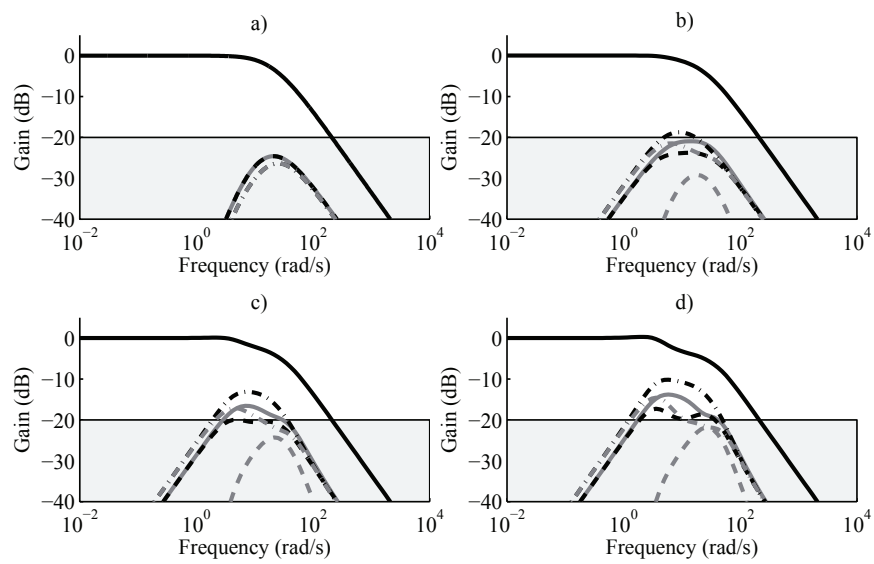

Speed: a) $0 \min ^{-1}$, b) $1 / 3 \omega_{n} \min ^{-1}$, c) $2 / 3 \omega_{n} \min ^{-1}$, d) $\omega_{n} \min ^{-1}$.

Fig. 8. Closed loop transfer function matrix Bode diagram representation at different rotational speeds. Input: stator $1 q$ axis current set-point. Outputs: machine currents in $q d$ reference. Closed loop time response design: $50 \mathrm{~ms}$

Fig. 7 shows the closed loop Bode diagram for different rotational speeds between $i_{s 1}^{q *}$ and all the machine currents $i_{s 1}^{q}$ for a closed loop time constant of $100 \mathrm{~ms}$. The curve relating $i_{s 1}^{q *}$ and $i_{s 1}^{q}$ presents $0 \mathrm{~dB}$ at zero frequency, which implies that the local controller is able to track current references. Besides, the curve relating the set-point $i_{s 1}^{q *}$ and the current $i_{s 1}^{d}$ presents reduced gains at all rotational speeds, fact that validates the decoupling loop action. However, it is observed that at non-zero rotational speeds, the curve relating stator $1 q$ axis set-point $i_{s 1}^{q *}$ and the $q$ and $d$ currents of the other stators show large gains, even exceeding the gain limitation imposed of $-20 \mathrm{~dB}$, fact that discards this PI settings for the machine current control.

Analogous Bode diagrams are depicted in Figs. 8 and 9 for closed loop time response constants of 50 and 20 ms respectively. Both figures show that the coupling gains between stator one and the others have been reduced, but they are still exceeding the gain limitation. From these graphs it can be concluded that faster controllers present a better control performance, reducing level of coupling between stators. This fact is reasonable, because faster local controllers allow to rapidly compensate the current deviations caused by current changes in other stators.

Finally, Fig. 10 shows the $\mathbf{T}$ matrix frequency response setting the closed loop time response to $10 \mathrm{~ms}$. It can be seen that the gain curve relating the $q$ set-point and current flowing through the $q$ axis of the stator one $\left(i_{s 1}^{q *}\right.$ and $\left.i_{s 1}^{q}\right)$, presents 0 dB 

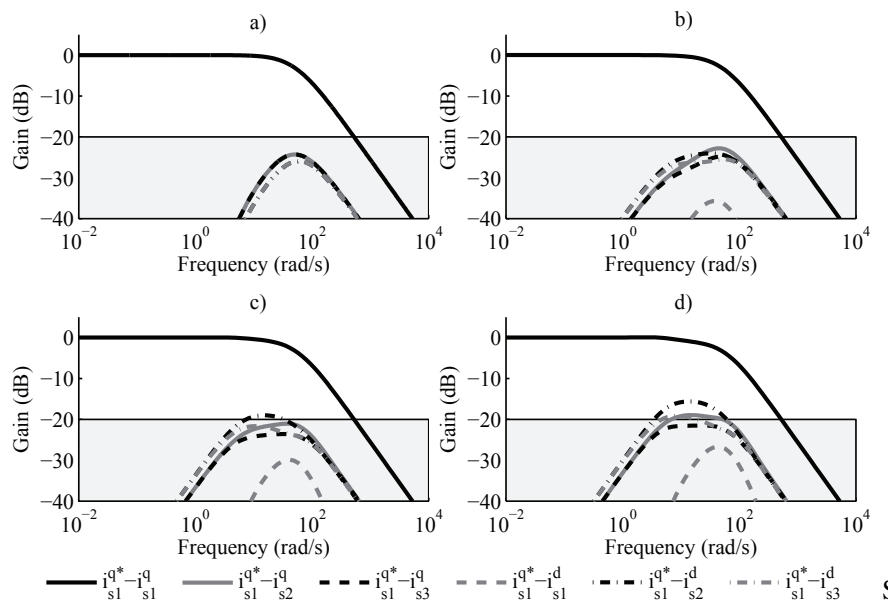

Speed: a) $0 \min ^{-1}$, b) $1 / 3 \omega_{n} \min ^{-1}$, c) $2 / 3 \omega_{n} \min ^{-1}$, d) $\omega_{n} \min ^{-1}$.

Fig. 9. Closed loop transfer function matrix Bode diagram representation at different rotational speeds. Input: stator $1 q$ axis current set-point. Outputs: machine currents in $q d$ reference. Closed loop time response design: $20 \mathrm{~ms}$
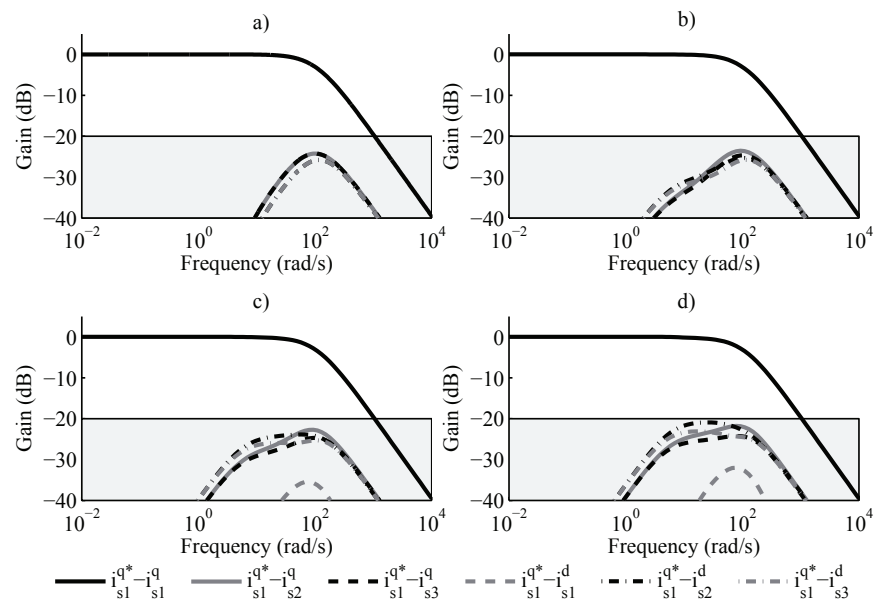

Fig. 10. Closed loop transfer function matrix Bode diagram representation at different rotational speeds. Input: stator $1 q$ axis current set-point. Outputs: machine currents in $q d$ reference. Closed loop time response design: $10 \mathrm{~ms}$

gain for constant reference inputs. Then, the controller involving these two variables is able to perfectly track constant current references. Besides, the gain curves related with the interactions between controllers do not exceed the established limitation. Then, it can be concluded that this controller is suitable for the machine because it accomplishes the defined control objectives.

It should also be considered that there is a maximum allowed bandwidth for the current loop. Typically, for classical converters, the fastest allowed time response should be at least 10 times slower than the switching period of the converter. Wind turbine converters, usually present operational switching frequencies in the low kilohertz range (1 to $5 \mathrm{kHz}$ ). Therefore, assuming a $2 \mathrm{kHz}$ of switching frequency, the fastest allowed closed loop time response should be larger than $5 \mathrm{~ms}$.

\section{Control validation}

In this section, the previously designed controller is validated through time domain simulations of the machine carried out in Matlab Simulink ${ }^{\circledR}$. To evaluate the current controller performance, two different case studies are prepared. In both cases, the simulation consists of introducing a step change to the stator $1 q$ axis current set-point $i_{s 1}^{q *}$ while maintaining the other current references set to zero, in order to validate the performance of the decentralized controller. This simulation is repeated at two different machine speeds to observe the influence of the speed to the controller performance.

The frequency design analysis performed in the previous section, has concluded that $10 \mathrm{~ms}$ was a suitable closed loop time response for the system. However, these simulations include also the other analyzed controllers (controller time response: 100, 50 and $20 \mathrm{~ms}$ ), aiming to validate the conclusions obtained during the design stage.

\section{A. Current set-point step change at rated speed}

This experiment is performed at the machine rated speed. A step change of $500 \mathrm{~A}$ is applied to the stator $1 q$ axis current set-point $i_{s 1}^{q *}$. Simulation results are shown in Fig. 11a.

The designed controller $(\tau=10 \mathrm{~ms})$ shows a good performance during the current step change. The current $i_{s 1}^{q}$ tracks the reference with a first order system behavior, reaching the $63 \%$ of the final value in approximately $10 \mathrm{~ms}$, validating 
a)
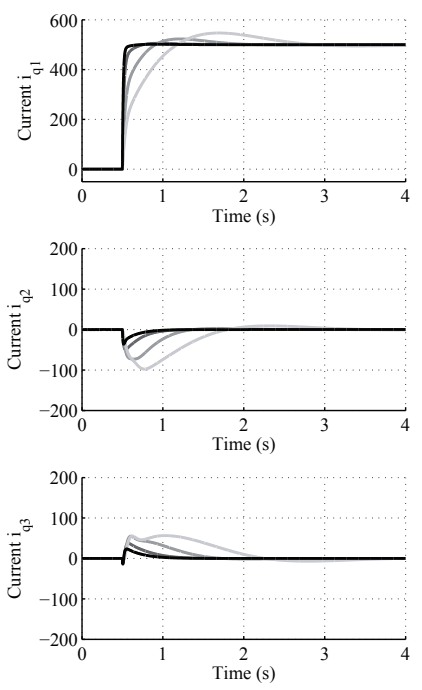
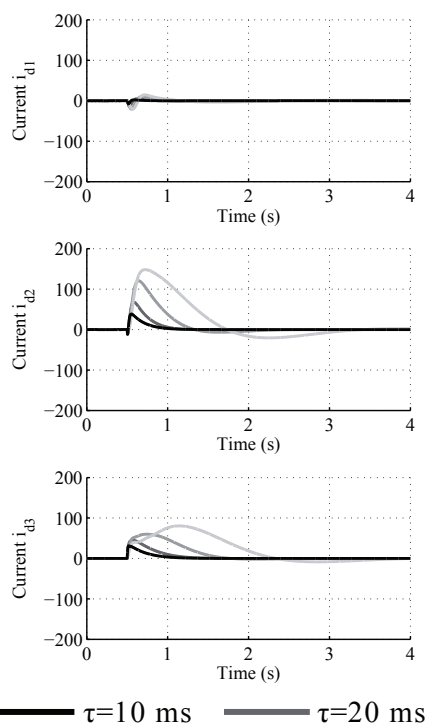

b)
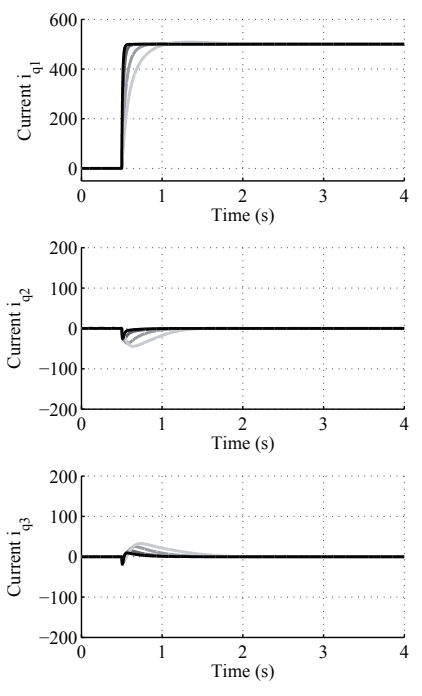

$\tau=50 \mathrm{~ms}=100 \mathrm{~ms}$
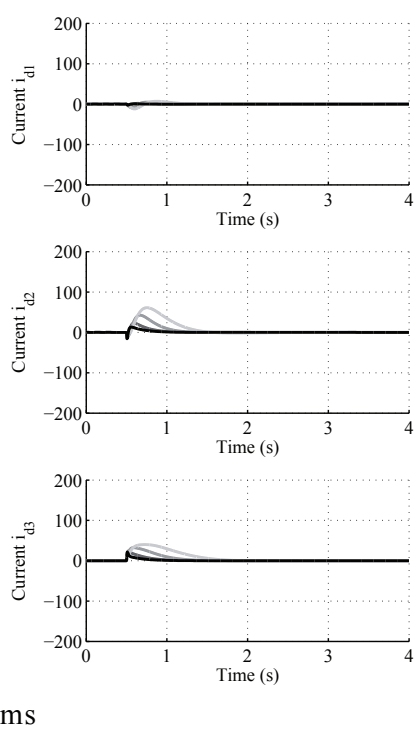

Fig. 11. Simulation of a set-point $q$ of the stator $1 i_{s 1}^{q *}$ step change, for different controller designs. Rotational speed: (a) Rated speed, (b) $1 / 3$ of rated speed

the IMC controller design. Besides, the current $d$ of the stator 1 is maintained near zero during the step change, due to the decoupling loop. It can also be seen that although the effects between the different stators are present, the current deviations are compensated in a few milliseconds by their respective local controllers, never exceeding the $10 \%$ of the set-point step amplitude (50 A).

Analyzing the temporal response of the slower controllers, it can be seen that all are able to track the current reference. However, after the reference step change, current deviations larger than the maximum established deviation appear (10\% of the set-point change $\Delta i_{s 1}^{q *}$ ). These results are consistent with the analysis performed in the control design stage.

\section{B. Current set-point step change at reduced speed}

The same experiment is performed at one third of the rated speed. Again, a step change of $500 \mathrm{~A}$ is applied to the stator 1 $q$ axis current set-point $i_{s 1}^{q *}$. Simulation results are shown in Fig. $11 \mathrm{~b}$.

This simulation shows a good performance of the designed controller $(\tau=10 \mathrm{~ms})$, even better than the previous one. It is also observed that the step change in the stator $1 q$ axis current set-point $i_{s 1}^{q *}$, affects the other stators less than at higher speeds. These results confirm that, as the machine speed increases, the coupling effects between the variables are more difficult to compensate.

It should be considered that in the previously presented simulations, the current set-points are changed in a stepwise manner. In real applications, reference changes should be ramp limited, because fast variations of these variables ( $q$ axis currents in this case) could imply fast fluctuations of the machine torque that may cause mechanical problems on the wind turbine.

\section{SCALED WIND TURBINE TEST RIG}

Once the methodology for designing the decentralized control is tested in simulation, the control algorithms are validated in a scaled wind turbine test rig. This rig is composed by two $30 \mathrm{~kW}$ PMSM, one acting as a motor and the other as a generator, mechanically connected through their shafts. The motor is a conventional three-phase machine connected to a standard frequency converter. The generator is a nine-phase machine with three independent star-connected stators, connected to the grid by means of three independent back-to-back converters. Fig. 12 shows the aforementioned machines, the cabinet where the frequency converter and the back-to-back converters for controlling the multi-phase machine are enclosed and a complete conceptual diagram of the setup.

The system is operated as follows: the motor acts as a wind emulator, using the frequency converter to regulate the speed of its shaft. The generator regulates the machine currents by means of the designed decentralized controller. The grid side converters carry out a voltage control of the DC bus to inject the active power coming from the generator to the grid.

Regarding the generator control, the decentralized control strategy has been implemented following the steps presented above. First, the machine model has been obtained based on the parameters from a FEM simulation, together with experimental tests developed with the machine. Then, applying the presented control design methodology, six PI current controllers (two for each stator) have been obtained and implemented on each of the DSP control boards of the back-to-back converters. The closed loop time response obtained from the study is $60 \mathrm{~ms}$, for each stator controller. Fig. 13 shows the transient response of the 


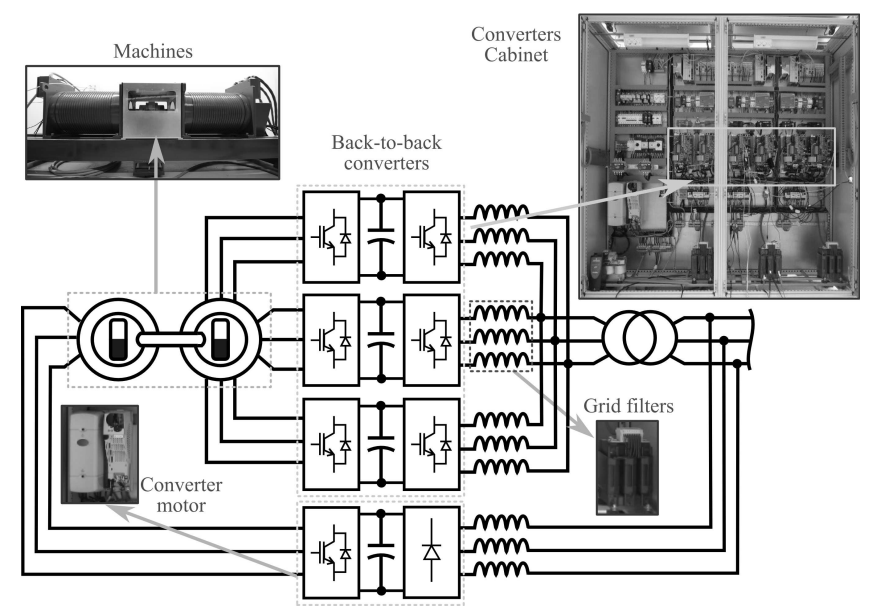

Fig. 12. Scaled wind turbine test rig photographs and conceptual drawing

decentralized controller for an active step change of the stator one current $i_{s 1}^{q *}$ while maintaining the other current set-points at zero, for three different speeds. It can be seen that the interaction between stators is reduced, even at high speeds. Besides, the current $a$ of the stator one shows a first order response with a time constant of approximately 60 ms, as chosen in the design stage. This test ensures that the decentralized controller implemented is able to regulate the machine currents without large interactions among stators during current transients.

a)

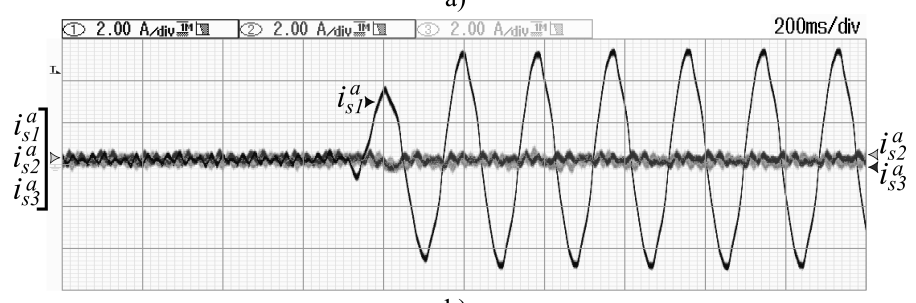

b)

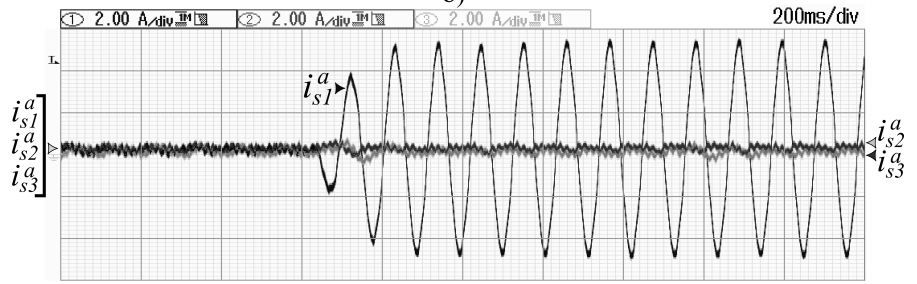

c)

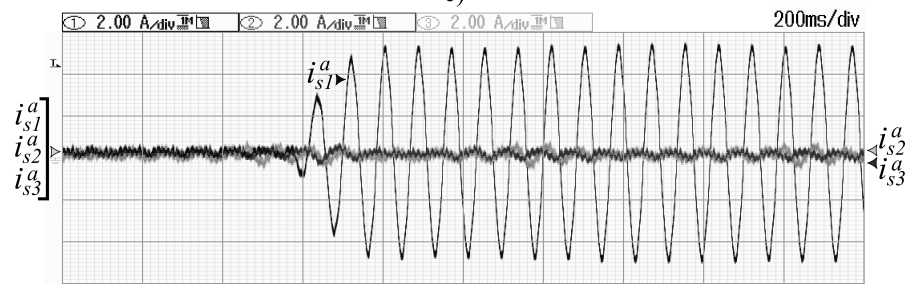

Fig. 13. Step change of the current $q$ set-point of the stator $1 i_{s 1}^{q *}$ for the decentralized controller designed, at different mechanical rotational speeds: a) 40 $\min ^{-1}$, b) $70 \min ^{-1}$, c) $90 \mathrm{~min}^{-1}$ (rated mechanical speed). Machine side converter phase $a$ stator currents.

Fig. 14a shows the experimental results of the decentralized control operation while extracting the same amount of active power from each of the stators. The oscilloscope capture shows that the currents flowing through the phase $a$ of each stator are identical, according to the power extraction scenario. Besides, a phase shift between the currents is observed caused by the electrical phase displacement $\left(40^{\circ}\right)$ existing between the stator winding sets. Fig. $14 \mathrm{~b}$ shows the grid side converter variables during the same experiment. It can be seen that the DC bus voltage is maintained at a constant value, by means of the DC voltage regulator, injecting the incoming power from the generator to the grid. This capture (Fig. 14b) also shows that the three phase $a$ currents are in phase, because these converters are all connected to the same AC grid .

Fig. 15 shows the results of a second experiment, in which the decentralized control is employed to extract a different 
a)

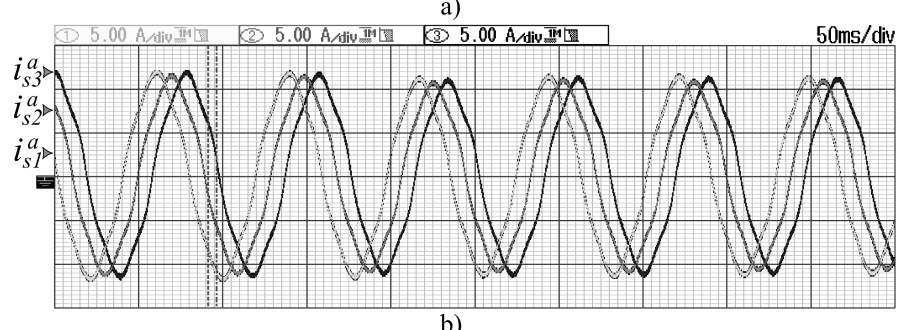

b)

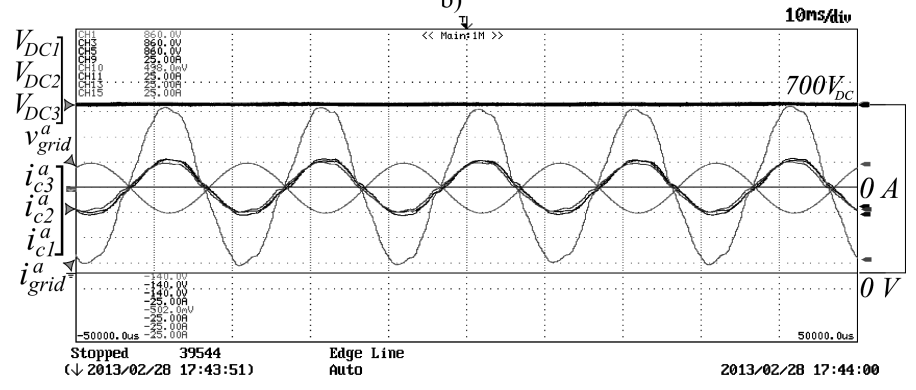

Fig. 14. Three converters extracting the same amount of power. a) Machine side converter phase $a$ stator currents. b) Grid side converter variables. AC currents scale: $5 \mathrm{~A} / \mathrm{div}$

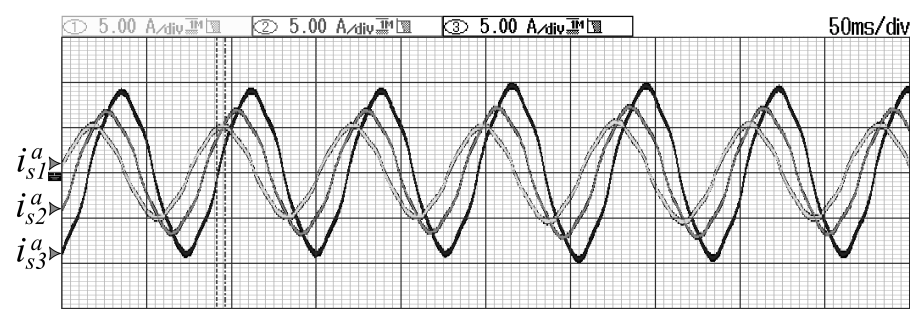

Fig. 15. Three converters extracting a different amount of power. Machine side converter phase $a$ stator currents.

amount of active power from each of the stators. It can be observed that the controller is able to regulate different active current set-points for each stator. Again, the phase shift between the machine phase $a$ currents is present.

\section{CONCLUSION}

The decentralized control design of a triple three-phase wind turbine generator is presented. The control is performed by three independent back-to-back converters connected to each of the generator stators. Each converter carries out its own vector control without sharing information with the rest of the converters. A complete control design methodology based on the classical vector control, combined with frequency analysis of the closed loop controller is developed. The control design methodology is applied to a nine-phase generator, tested in simulation and validated in a scaled wind turbine generator test rig. The performed tests have shown satisfactory results proving the concept viability. These results confirm that this generator configuration combined with the designed controllers could be an interesting alternative for offshore applications, showing additional capabilities in terms of redundancy and control possibilities.

\section{REFERENCES}

[1] The European offshore wind industry - key trends and statistics 2013. European Wind Energy Association, EWEA, 2013.

[2] Europe's onshore and offshore wind energy potential. Eur. Environmental Agency, Copenhaguen, 2009.

[3] Wind Energy Technology Roadmap. Energy Agency, Paris, 2013.

[4] S. Faulstich, B. Hahn, and P. J. Tavner, "Wind turbine downtime and its importance for offshore deployment," Wind Energy, vol. 14, no. 3, pp. 327-337, 2011.

[5] R. Semken, M. Polikarpova, P. Roytta, J. Alexandrova, J. Pyrhonen, J. Nerg, A. Mikkola, and J. Backman, "Direct-drive permanent magnet generators for high-power wind turbines: benefits and limiting factors," IET Renewable Power Generation, vol. 6, no. 1, pp. 1-8, January 2012.

[6] S. Brisset, D. Vizireanu, and P. Brochet, "Design and optimization of a nine-phase axial-flux pm synchronous generator with concentrated winding for direct-drive wind turbine," IEEE Trans. Ind. Appl., vol. 44, no. 3, pp. 707-715, May 2008.

[7] J. Sauter and A. Mirzaian, "Encoderless control of a 6mw permanent magnet synchronous generator," in 15th Eur. Conf. on Power Electron. and Appl. (EPE), 2013, Sept 2013, pp. 1-10.

[8] E. Prieto-Araujo, A. Junyent-Ferre, D. Lavernia, and O. Gomis-Bellmunt, "Distributed control of a nine phase permanent magnet generator and scaled platform validation," in EWEA 2014, March 2014.

[9] E. Levi, "Multiphase electric machines for variable-speed applications," IEEE Trans. Ind. Electron., vol. 55, no. 5, pp. 1893-1909, May 2008.

[10] R. Bojoi, M. Lazzari, F. Profumo, and A. Tenconi, "Digital field-oriented control for dual three-phase induction motor drives," IEEE Trans. Ind. Appl., vol. 39, no. 3, pp. 752-760, May 2003. 
[11] L. De Camillis, M. Matuonto, A. Monti, and A. Vignati, "Optimizing current control performance in double winding asynchronous motors in large power inverter drives," IEEE Trans. Power Electron., vol. 16, no. 5, pp. 676-685, Sep 2001.

[12] J. Karttunen, S. Kallio, P. Peltoniemi, P. Silventoinen, and O. Pyrhonen, "Decoupled vector control scheme for dual three-phase permanent magnet synchronous machines," IEEE Trans. Ind. Electron., vol. 61, no. 5, pp. 2185-2196, May 2014.

[13] L. Hua, Z. Yunping, and H. Bi, "The vector control strategies for multiphase synchronous motor drive systems," in IEEE Int. Symp. on Ind. Electron., 2006, vol. 3, July 2006, pp. 2205-2210.

[14] P. Krause, Analysis of electric machinery, ser. McGraw-Hill series in Elect. and Comput. Eng. McGraw-Hill, 1986.

[15] T. Lipo, "A dq model for six phase induction machines," in Int. Conf. on Electric Machines, Athens, Greece, 1980, pp. 860-867.

[16] Y. Zhao and T. Lipo, "Space vector pwm control of dual three-phase induction machine using vector space decomposition," IEEE Trans. Ind. Appl., vol. 31, no. 5, pp. 1100-1109, Sep 1995 .

[17] M. Andriollo, G. Bettanini, G. Martinelli, A. Morini, and A. Tortella, "Analysis of double-star permanent-magnet synchronous generators by a general decoupled d-q model," IEEE Trans. Ind. Appl., vol. 45, no. 4, pp. 1416-1424, July 2009.

[18] S. Kallio, M. Andriollo, A. Tortella, and J. Karttunen, "Decoupled d-q model of double-star interior-permanent-magnet synchronous machines," IEEE Trans. Ind. Electron., vol. 60, no. 6, pp. 2486-2494, June 2013.

[19] M. Duran, S. Kouro, B. Wu, E. Levi, F. Barrero, and S. Alepuz, "Six-phase pmsg wind energy conversion system based on medium-voltage multilevel converter," in Proc. of the 2011-14th Eur. Conf. on Power Electron. and Appl. (EPE 2011), Aug 2011, pp. 1-10.

[20] T. Lipo, Analysis of Synchronous Machines, Second Edition. Taylor \& Francis, 2012.

[21] J. Hendershot and T. Miller, Design of Brushless Permanent Magnet Motors, ser. McGraw-Hill series in Elect. and Comput. Eng., 2010.

[22] C. Ong, Dynamic Simulation of Electric Machinery: Using MATLAB/SIMULINK. Prentice Hall PTR, 1998.

[23] R. Nelson and P. Krause, "Induction machine analysis for arbitrary displacement between multiple winding sets," IEEE Trans. Power App. Syst., vol. PAS-93, no. 3, pp. 841-848, May 1974.

[24] L. Harnefors and H.-P. Nee, "Model-based current control of ac machines using the internal model control method," IEEE Trans. Ind. Appl., vol. 34, no. 1, pp. 133-141, Jan 1998.

[25] S. Skogestad and I. Postlethwaite, Multivariable feedback control: analysis and design. Wiley, 1996.

[26] S.-K. Chung, "A phase tracking system for three phase utility interface inverters," IEEE Trans. on Power Electron., vol. 15, no. 3, pp. 431-438, 2000. 